\title{
DINÁMICA DE LA RADIACIÓN FOTOSINTÉTICAMENTE ACTIVA EN ARREGLOS AGROFORESTALES CON Hevea brasiliensis EN EL NORTE DE LA AMAZONIA COLOMBIANA
}

\section{DYNAMIC OF THE PHOTOSYNTHETICALLY ACTIVE RADIATION IN ARRANGEMENTS AGROFORESTALES WITH Hevea brasiliensis IN NORTHERN COLOMBIA AMAZON}

\author{
Ervin Humprey Durán-Bautista ${ }^{1}$, Yimi Katherine Ángel-Sánchez² ${ }^{2}$ Juan Carlos Suárez-Salazar ${ }^{3}$
}

\begin{abstract}
${ }^{1}$ Ingeniero Agroecólogo, Magister en Agroforestería, Profesor Universidad de la Amazonia, Facultad de Ingeniería, Programa Ingeniería Agroecológica. Sede Principal, calle 17 Diag. 17 Cra. 3F Barrio Porvenir, Florencia-Caquetá, e-mail: e.duran@ udla.edu.co; ${ }^{2}$ Ingeniero Agroecólogo, Magister en Agroforestería. Universidad de la Amazonia. Sede Principal, calle 17 Diag. 17 Cra. 3F Barrio Porvenir, Florencia-Caquetá, e-mail: kangel0529@gmail.com; ${ }^{3}$ Ingeniero Agroecólogo, M.Sc. Profesor Universidad de la Amazonia, Facultad de Ingeniería, Programa Ingeniería Agroecológica. Sede Principal, calle 17 Diag. 17 Cra. 3F Barrio Porvenir, Florencia-Caquetá, e-mail: ju.suarez@udla.edu.co
\end{abstract}

Rev. U.D.C.A Act. \& Div. Cient. 18(2): 365-372, Julio-Diciembre, 2015

\section{RESUMEN}

Los arreglos agroforestales con caucho (Hevea brasiliensis (Willd. ex A. Juss.) Müll. Arg.) en el departamento del Caquetá, Colombia, son importantes para la economía campesina y cuenta con una área sembrada de 6.017 ha, por ello, se evaluó la influencia de la estructura del arreglo agroforestal de caucho asociado con cacao (Theobroma cacao L.) y copoazú (Theobroma grandiflorum (Willd. ex Spreng.) K. Schum.) sobre la distribución de la radiación fotosintéticamente activa transmitida (fPARt), en lotes ubicados en el municipio de Morelia, al sur occidente del departamento. Se seleccionaron lotes de diferentes edades y especies. Se realizó el muestreo de la radiación transmitida en un área de $2.500 \mathrm{~m}^{2}$, cuya información fue modelada, utilizando el programa estadístico $\mathrm{R}$ 3.1.2, a través del paquete akima. Para la estructura agroforestal, se empleó el simulador forestal SEXi - FS. Los resultados muestran un efecto de la edad del lote sobre la radiación transmitida $(\mathrm{p}<0,0001)$, así como una distribución irregular de los individuos al interior del arreglo; como consecuencia de ello, la fPARt no está distribuida homogéneamente, generando áreas donde la radiación transmitida es alta y otras, donde es baja. Esta situación plantea la necesidad de mejorar el manejo y la distribución de las especies al interior de los arreglos agroforestales de caucho, para hacerlos más eficientes, en el uso y la distribución de la radiación.
Palabras clave: Sombra, distribución espacial, dosel, caucho natural.

\section{SUMMARY}

Agroforestry arrangements with rubber (Hevea brasiliensis (Willd. Ex A. Juss.) Müll. Arg.), at the department of Caquetá (Colombia), with a planted area of 6017ha, are important for the rural economy. Therefore, the influence of the rubber agroforestry structure, associated with cocoa (Theobroma cacao L.) and copoazú (Theobroma grandiflorum (Willd. ex Spreng.) K. Schum.) on the distribution of the transmitted photosynthetically active radiation (fPart) in plots, located in the municipality of Morelia in the western southern region of the department was evaluated. Plots of different ages and species were selected. Sampling of the transmitted radiation in an area of $2500 \mathrm{~m}^{2}$ was made, and the information modeled using the statistical software R 3.1.2, through the akima package. For the agroforestry structure the Sexi FS simulator was employed. The results revealed an effect of plots age on the transmitted radiation ( $p<0.0001)$, as well as an irregular distribution of the individuals within the arrangement. As consequence, the fPARt is not homogeneously distributed, generating areas where the transmitted radiation is high and others where it is low. This raises the need to improve the management and distribution of species within agroforestry arrangements with rubber, to make them more efficient in the use and distribution of radiation 
Key words: Shade, spatial distribution, canopy, natural rubber.

\section{INTRODUCCIÓN}

La radiación solar es la principal fuente de energía para la fotosíntesis y juega un papel importante en el crecimiento y en el desarrollo vegetal, especialmente, por su cantidad y su distribución espectral (Farfán et al. 2003). La radiación fotosintéticamente activa, se encuentra en una longitud de ondas visibles, entre 400 y $700 \mu \mathrm{m}$, la cual, es capturada y almacenada por las plantas, a través de sus sistemas fotosintéticos (Tsubo \& Walker, 2005).

La predicción de las variaciones temporales y espaciales de la intensidad de la radiación en el estrato arbóreo es importante, para definir densidades de siembra de especies, que serán ubicadas en el estrato inferior del arreglo (Talbot \& Dupraz, 2012). Por ello, se han desarrollado modelos ecofisiológicos, para simular el crecimiento y la productividad, a partir de la PAR, debido a la incidencia en procesos, como la fotosíntesis (Wilson \& Meyers, 2007); sin embargo, los valores de PAR utilizados en estos modelos, se derivan, típicamente, de un pequeño número de estudios empíricos, debido a que esta variable no es evaluada rutinariamente (Wu et al. 2010).

Esta falta de datos derivados empíricamente significa que la PAR es estimada como una fracción constante de la banda ancha de la irradiancia solar (Rs), frecuentemente, entre 35 a 50\%, dependiendo del tiempo y las condiciones climáticas (Yunlin \& Boqiang, 2002). Este método está sujeto a errores derivados de la influencia de temporal climático y variaciones espaciales de la relación entre PAR y Rs (Zhang et al. 2000). Otro aspecto que se debe considerar es el diseño no apropiado de los arreglos agroforestales para proveer de sombra a los cultivos del dosel inferior; la PAR se presenta de manera heterogénea, generando parches muy sombreados y otros muy expuestos al sol, afectando el crecimiento y el desarrollo de los cultivos. Ejemplo de ello, son los bajos rendimientos obtenidos en ciertos cultivos, como café y cacao, cuando la sombra es mayor al $50 \%$, superando los requerimientos fisiológicos (DaMatta \& Rodríguez, 2007) e inhibiendo el desarrollo de las plantas, ya que se modifica el régimen de la radiación -fotosintéticamente activa-, incidente en calidad y en cantidad (Somarriba \& Beer, 2011). Particularmente, en especies leñosas perennes de estrato bajo, como el cacao, un exceso de radiación, por arriba de los 700 (umol m $\mathrm{s}^{-1}$ ), puede ocasionar efectos fotoinhibitorios temporales o permanentes, de acuerdo a los mecanismos de fotoprotección, que posee la especie (Almeida et al. 2014).

El cultivo del caucho en el departamento del Caquetá es importante para la economía campesina, debido al ingreso por venta de material coagulado, obtenido del látex, en un área sembrada de 6.017ha, dada su importancia económica y los avances en investigaciones sobre las ventajas comparativas de los sistemas agroforestales, frente a las plantaciones en monocultivo, en cuanto a crecimiento, producción y disminución de plagas y enfermedades; en la región, se ha venido promoviendo la conversión de las plantaciones a arreglos agroforestales, donde se asocian con cultivos, como cacao, frutales amazónicos, maderables y bancos de proteína (Sterling et al. 2014). Se estudió el arreglo agroforestal de caucho - cacao y cómo influye su estructura sobre la distribución de la radiación fotosintéticamente activa. En este sentido, se esperaría que existan diferencias entre las estructuras agroforestales evaluadas, con el fin de generar aportes en el diseño y en el mejoramiento de estos sistemas productivos, teniendo en cuenta las condiciones climáticas de la región amazónica.

\section{MATERIALES Y MÉTODOS}

Área de estudio. Los arreglos agroforestales (Cuadro 1), se localizaron en el municipio de Morelia, al sur occidente del departamento del Caquetá, a una distancia de $24 \mathrm{~km}$ del municipio de Florencia, ubicado a $1^{\circ} 29^{\prime} 25^{\prime \prime}$ N y $75^{\circ} 43^{\prime} 43^{\prime \prime}$ $\mathrm{O}$, a una altitud de $258 \mathrm{~m}$. El área presenta una precipitación anual promedia de $3.493 \mathrm{~mm}$, un brillo solar de 4,1 horas día $^{-1}$, temperatura promedia de $24,4^{\circ} \mathrm{C}$ y humedad relativa promedia de $85,9 \%$.

Diseño experimental. El modelo experimental corresponde a un diseño completamente al azar, con tres tratamientos, que corresponden a los rangos de edad del cultivo y cuatro repeticiones.

Cuantificación de la radiación fotosintéticamente activa. En cada arreglo agroforestal $(n=12)$, se ubicaron parcelas de $50 \times 50 \mathrm{~m}$, donde se registró cada metro, mediante el uso de un Ceptómetro AccuPAR $®$ LP-80 (Decagon devices USA); la radiación fotosintéticamente activa transmitida (fPARt), ubicando la barra cuántica bajo el dosel; la radiación fotosintéticamente activa incidente (PAR), se determinó con un sensor externo, que se ubicó en un área a plena exposición solar sin presencia de árboles, ni objetos que intervinieran en la medición y, la radiación fotosintéticamente activa interceptada (fPARi), se calculó por diferencia entre la PAR y la fPARt. Todas las mediciones fueron realizadas entre las 11:00 y 13:00 horas de días completamente despejados, lapso en que se encuentra la mayor cantidad de flujo fotosintético de fotones y en la época de mínima precipitación, evitando la interferencia e incidencia de la nubosidad.

Medición de variables dasométricas. A cada uno de los árboles de caucho, se le determinó las variables dimensionales, como diámetro a la altura del pecho (dap), altura total (ht), altura de la copa (hc), diámetro de copa y el área de copa (ac). El dap, se midió con cinta métrica que, 
Cuadro 1. Arreglos agroforestales de caucho evaluados en la amazonia colombiana.

\begin{tabular}{|l|l|}
\hline \multicolumn{1}{|c|}{ Edad } & \multicolumn{1}{c|}{ Descripción } \\
\hline $\mathbf{1}$ a $\mathbf{7}$ años & $\begin{array}{l}\text { Arreglo agroforestal de caucho (Hevea brasiliensis (Willd. ex A.Juss.) Müll.Arg.) } \\
\text { diseñado en surcos dobles de 3x3 y 7m entre surcos, espacio en el que se asocia } \\
\text { con cacao (Theobroma cacao L.) }\end{array}$ \\
\hline $\mathbf{7}$ a $\mathbf{1 5}$ años & $\begin{array}{l}\text { Arreglo agroforestal con caucho natural (Hevea brasiliensis) en la cohorte } \\
\text { superior, asociado con cacao (Theobroma cacao L.) y Marfil (Siparuna decipiens } \\
\text { Tul. A.DC.) }\end{array}$ \\
\hline mayor a $\mathbf{1 5}$ años & $\begin{array}{l}\text { Arreglo agroforestal compuesto por árboles de caucho (Hevea brasiliensis) } \\
\text { con frutales amazónicos Copoazú (Theobroma grandiflorum (Wild. ex Spring) } \\
\text { Schumann) y cacao (Theobroma cacao L.) }\end{array}$ \\
\hline
\end{tabular}

para este caso, se transformaron los valores de circunferencia al diámetro, empleando la constante circular; la altura total y del fuste, se calcularon con clinómetro y con cinta métrica; el diámetro de copa, se midió con cinta métrica, promediando dos mediciones perpendiculares de la proyección vertical de las copas sobre el suelo y, el área de copa, se estimó como el área de un círculo, cuyo diámetro (D) es el diámetro promedio de copa (Segura \& Andrade, 2007).

Modelamiento de la radiación fotosintéticamente activa y la estructura agroforestal. Para el modelamiento espacial de la PAR, se utilizó el programa estadístico de $R$ versión 3.1.2, a través del package akima, que emplea una lista con los componentes X, Y, Z, de los cuales, representa los valores de $Z$ en un plano biplot, a partir de puntos seleccionados irregularmente, distribuidos en el plano XY (Akima, 2015). Para la estructura agroforestal, se empleó el simulador forestal SEXi - FS, que se centra en las interacciones árbolárbol, de múltiples especies forestales. Este modelo aplica un enfoque orientado a objetos y brinda la posibilidad de hacer representaciones en tres dimensiones de una parcela, para este caso, de $2.500 \mathrm{~m}^{2}$, que sirve como base para la simulación. El objetivo principal de este modelo es conseguir una representación dinámica y coherente de un sistema complejo, donde la complejidad, se refiere aquí, a la interacción de individuos con diferentes propiedades más que a la complejidad de los procesos elementales que intervienen (Vincént et al. 2002).

Análisis estadístico. Para evaluar los datos de cada una de las variables radiación fotosintéticamente activa (PAR), la incidente (PARi), la transmitida (PARt) y la fracción de radiación interceptada (fPARi), se realizó un análisis de varianza (ANOVA), a través del paquete estadístico $R$ versión 3.1.2, basado en el package nlme para modelos lineales y no lineales de efectos mixtos, permitiendo efectos aleatorios anidados y se desarrollaron pruebas de significancia LSD de Fisher, para detectar diferencias entre medias.

\section{RESULTADOS Y DISCUSIÓN}

Se encontró un efecto de la edad de los arreglos agroforestales de caucho sobre la radiación transmitida al cultivo de cacao; en este sentido, se evidencia que a mayor edad del arreglo agroforestal es menor la fPARt (Figura 1); lo anterior, se debe a los cambios producidos con el crecimiento y el desarrollo de la planta de caucho sobre el área de copa, altura, arquitectura y cantidad de follaje; diferentes estudios muestran que la proporción de la biomasa del follaje cambia en función del tamaño del árbol (Acosta et al. 2002; Martínez et al. 2005). Kume et al. (2011) argumentan que los cambios constantes en el nivel de radiación, se deben a los cambios en la estructura del dosel.

Estos resultados recobran importancia, debido a que cambios en la cantidad y la calidad de la radiación trasmitida afectan significativamente los cultivos que crecen bajo la sombra del dosel. En este sentido, diferentes autores, entre ellos Righi et al. (2008) y Ourado-Neto \& Favarin (2008) plantean que las leñosas perennes presentan una clara variación sobre la arquitectura y el crecimiento vertical que se incrementa bajo la copa de árboles de caucho, de manera que estas plantas desarrollan una alta capacidad para interceptar y usar la radiación.

Los resultados muestran que los arreglos agroforestales mayores a 15 años de edad presentan una mayor fracción de radiación interceptada, por lo tanto, los valores de fPARt son más bajos; esto se debe, a que el dosel se comporta como un gran filtro óptico, compuesto de muchos microfiltros, lo que indica que las cantidades de PAR absorbidas por las láminas foliares se incrementan con el aumento del índice de área foliar (Tsubo et al. 2003), básicamente, por la acción del follaje que absorbe la radiación de manera selectiva y hace que la calidad de la radiación también cambie, considerablemente, dentro de la copa (Ninemets \& Anten, 2009). 


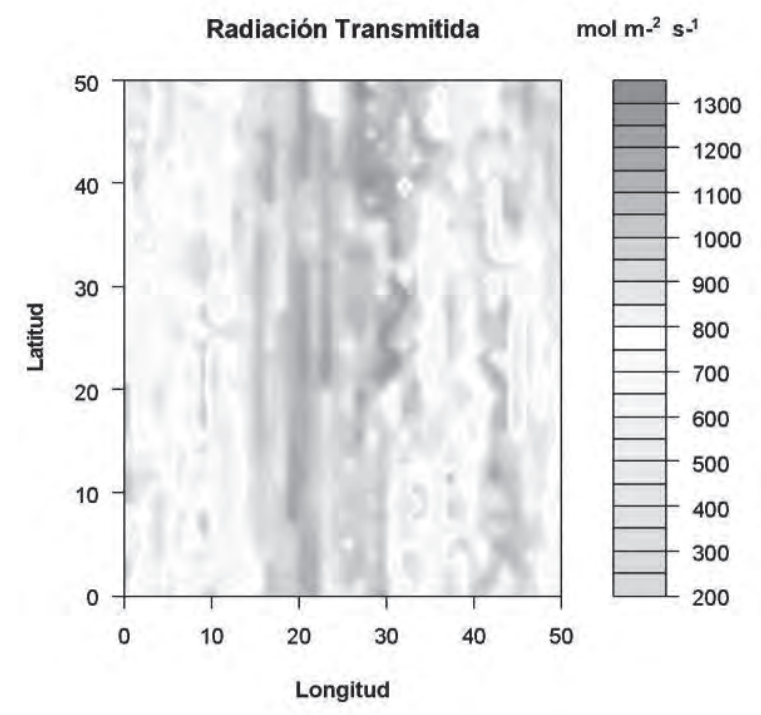

A) 7 a 15 años.

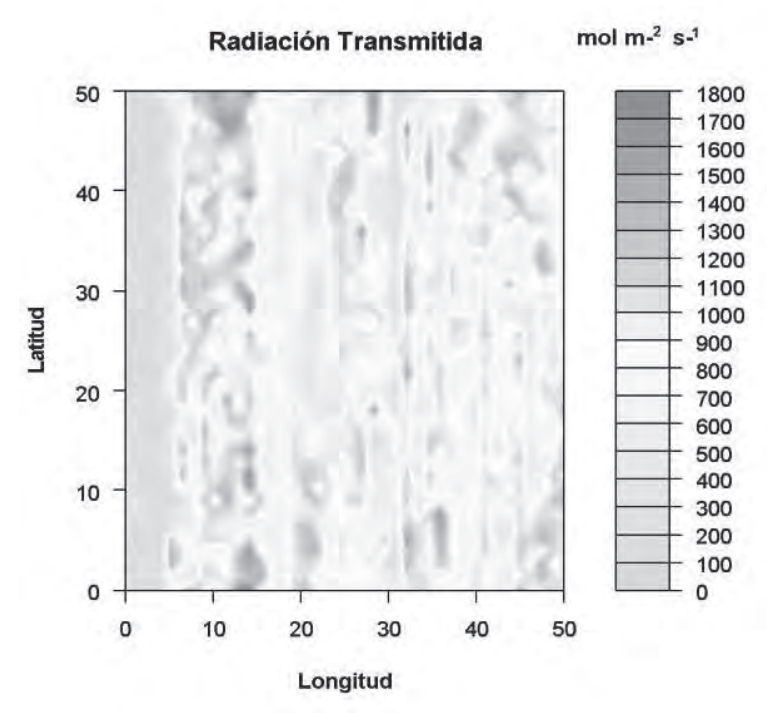

B) Mayores a 15 años.

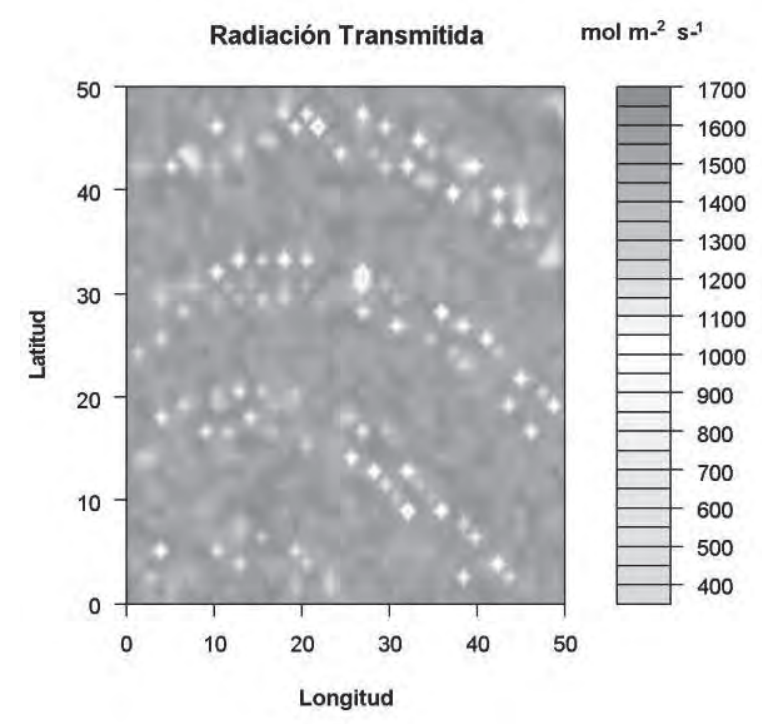

C) 1 a 7 años.

Figura 1. Distribución espacial de la PARt en arreglos agroforestales: A) entre 7 a 15 años; B) mayor a 15 años y C) entre 1 a 7 años de edad.

Entre los arreglos agroforestales, se presentaron diferencias significativas ( $P<0,0001)$, siendo mayor la radiación transmitida para los arreglos agroforestales cuyo rango de edad oscila entre uno a siete años, contrario a lo que se muestra en los arreglos agroforestales mayores a 15 años de edad, donde la fPARt es menor (Tabla 1).
La fPARt que se presenta en los arreglos agroforestales entre siete y 15 años y mayores 15 años de edad es adecuada, ya que las densidades de flujo fotónico son menores de $1000 \mu \mathrm{mol} \mathrm{m}{ }^{2} \mathrm{~s}^{-}{ }^{1}$, para evitar el daño fotoinhibitorio que se registra en cultivos de leñosas perennes, como el Theobroma cacao L. (Jaimez et al. 2008); esto repercute en 
Tabla 1. Medias de las variables evaluadas en los arreglos agroforestales con Hevea brasiliensis, de diferentes edades.

\begin{tabular}{|c|c|c|c|c|}
\hline Edad & \%PARi & Media PARi $\left(\mu \mathrm{mol} \mathrm{m}-^{2} \mathrm{~s}^{-1}\right)$ & \%PARt & Media PAR $\mathrm{t}\left(\mu \mathrm{mol} \mathrm{m-}{ }^{2} \mathrm{~s}^{-}\right)$ \\
\hline 1-7 años & $26,11 \pm 0,16 a$ & $467,81 \pm 3,21 a$ & $73,89 \pm 0,16 c$ & $1316,59 \pm 3,09 c$ \\
\hline 7 - 15 años & $44,18 \pm 0,16 b$ & $684,06 \pm 3,27 b$ & $55,82 \pm 0,18 b$ & $879,41 \pm 3,09 b$ \\
\hline$>15$ años & $47,52 \pm 0,18 c$ & $723,49 \pm 3,72 c$ & $52,48 \pm 0,16 a$ & $769,75 \pm 3,57 a$ \\
\hline
\end{tabular}

Medias seguidas de diferentes letras en la columna son estadísticamente diferentes, según la prueba de LSD Fisher.

la menor duración y respiración de las hojas y, en algunos momentos del año, a problemas de déficit hídricos (Jaimez et al. 2008). En cambio, en cultivos de café bajo sombra, la fPARt promedio obtenida para los arreglos agroforestales de todas las edades excede los límites de saturación, puesto que, en general, los cafetales de sombra requieren entre $60 \mathrm{y}$ $200 \mu \mathrm{mol} \mathrm{m-}{ }^{2}$ s- $^{1}$ (Azcón et al. 2008; DaMatta, 2004).

Estos resultados se ven influenciados por el estado de desarrollo del cultivo; en este sentido, Lindquist et al. (2005) mencionan que la materia seca producida y la calidad de la producción muestra una correlación positiva con la cantidad de radiación interceptada por las plantas. En consecuencia, los resultados obtenidos de mayor interceptación de la radiación en los cultivos de mayor edad, probablemente, estén relacionados, de manera directa, con un mayor desarrollo de biomasa, que se vería reflejado en una menor fPARt.

Por otra parte, se plantea que la estructura aérea de la planta está determinada por el tamaño, la forma, la orientación, la distribución y la posición de los diferentes órganos: hojas, tallos, ramas, flores y fruto (Jaramillo et al. 2006); asimismo,
Farfán et al. (2003) sostienen que, tanto la interceptación como la transmisión de la PAR, están determinadas por la arquitectura de la especie y la homogeneidad de la población; estas características cambian constantemente, de acuerdo a la tasa de crecimiento y del ciclo de vida de la especie, por lo tanto, difieren entre los arreglos agroforestales mayores a 15 años y los menores a siete años de edad.

En la figura 2, se muestra la distribución espacial de los árboles de caucho en los arreglos agroforestales, así como la simulación de los individuos, a partir de variables biométricas, como altura total y de copa, diámetro a la altura del pecho, diámetro y área de copa. En general, se encontró una distribución irregular de los individuos en los arreglos agroforestales, entre los rangos de siete a 15 y mayores a 15 años de edad, lo que se traduce en una distribución de la radiación transmitida de una manera heterogénea; esta situación pude estar asociada con problemas en la planificación agroforestal, al no realizar actividades de resiembra y, por otra parte, el arreglo agroforestal de uno a siete años presenta una distribución homogénea de la radiación, que responde a un modelo de plantación regular (Figura 3).

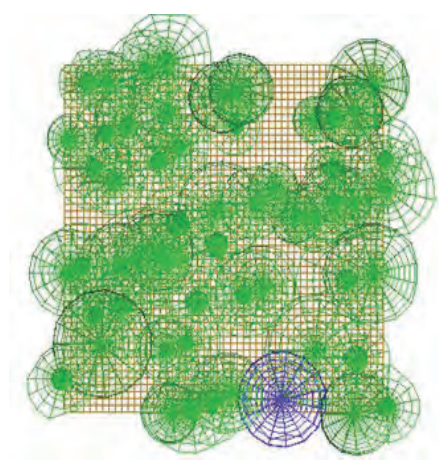

A) 7 a 15 años

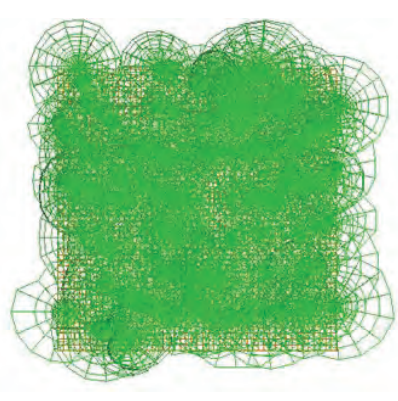

B) Mayores a 15 años

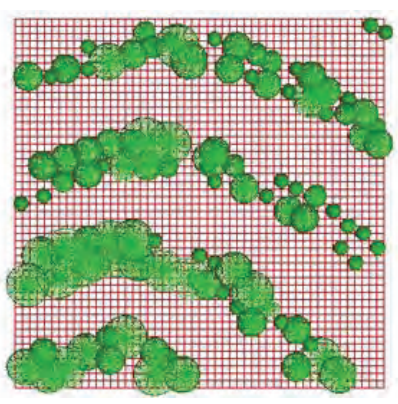

C) 1 a 7 años

Figura 2. Simulación en el Software SExI-FS®: A) distribución espacial de los individuos de Hevea brasiliensis, entre 7 a 15 años; B) distribución espacial de los individuos de Hevea brasiliensis, entre mayores a 15 años y C) distribución espacial de los individuos de Hevea brasiliensis, entre 1 y 7 años. 


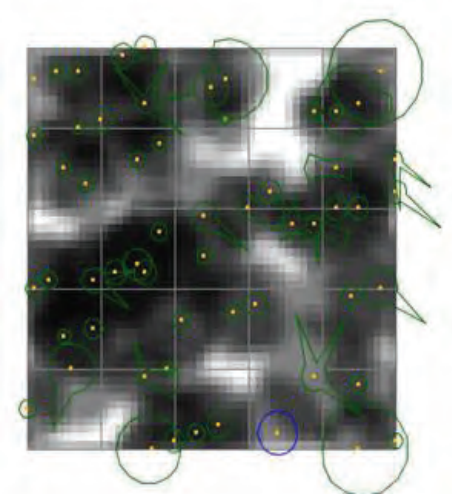

A) 7 a 15 años.

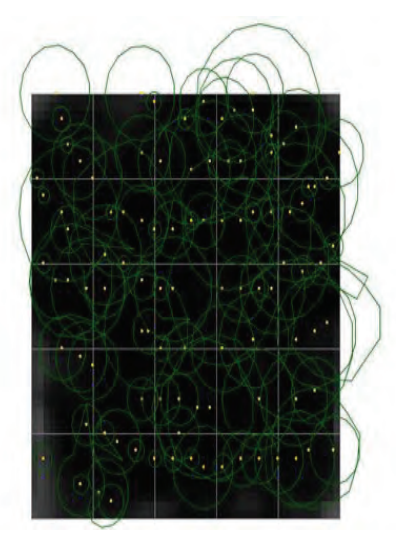

B) Mayores a 15 años

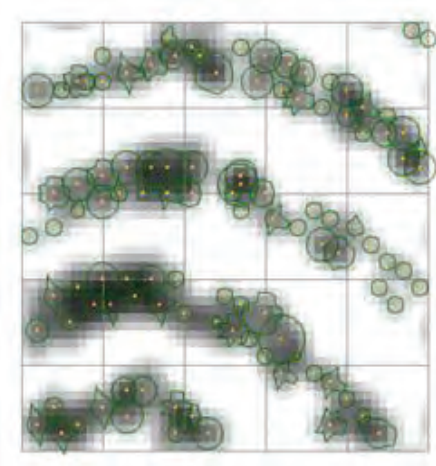

C) 1 a 7 años.

Figura 3. Simulación en el Software SExI-FS ${ }^{\circledR}$ : A) grises intensos muestran el área en el que la radiación interceptada es mayor en SAF, de 7 a 15 años; B) grises intensos muestran el área en el que la radiación interceptada es mayor en SAF, mayores a 15 años y C) colores intensos de grises muestran el área en el que la radiación interceptada es mayor en SAF, de 1 y 7 años.

Eneste sentido, diversos autores (Nocketal.2008; Greenwood et al. 2009) afirman que, además de las particularidades de la planta, como la edad y el tamaño, las características de la plantación, como la densidad de siembra y el marco de plantación del dosel, afectan la distribución de la radiación fotosintéticamente activa transmitida, constituyendo, a menudo, un factor limitante para el establecimiento, el crecimiento y el rendimiento de los cultivos asociados, situación que genera consecuencias importantes, por los cambios en el eficiente uso de la radiación.

Además, se muestra el comportamiento de la radiación, en el que las áreas que presentan grises intensos indican una mayor intensidad de la captación de la radiación o sombra. Esto tiene que ver con los atributos de la especie, como densidad del follaje y forma de la copa, así como de la estructura del dosel de sombra, es decir, la composición y la densidad de las especies arbóreas en el lote o parcela, que determinan la radiación solar que reciben las plantas de los estratos más bajos (Cerdán, 2007).

Asimismo, Somarriba \& Beer (2011) plantearon que las características del dosel de sombra -determinadas por la composición botánica, el número de capas verticales o subestratos, el grado de cobertura de dosel, las características de la copa del árbol y el manejo de la sombra del árbol, como la poda, el raleo y la reforestación-, varían mucho entre las principales zonas ecológicas, en respuesta a factores biofísicos. Al respecto, los resultados aquí presentados serán un insumo para la toma de decisiones de los productores de caucho de la región amazónica colombiana.
Finalmente, se concluye que la radiación fotosintéticamente activa interceptada y transmitida varía considerablemente en función de la edad de siembra y el estado de desarrollo de los arreglos agroforestales de caucho; de otra parte, existe una distribución irregular de los individuos al interior del arreglo, para los rango de edad de siete a 15 años y mayores de 15; como consecuencia de ello, la PAR no está distribuida homogéneamente, generando áreas donde la radiación transmitida es alta y otras donde es baja; esta situación plantea la necesidad de mejorar el manejo y la distribución de las especies al interior de los arreglos agroforestales de caucho, para hacerlos más eficientes en el uso y la distribución de la radiación, de manera que permita mejorar la productividad y la rentabilidad de los cultivos que crecen bajo sombra.

Agradecimiento: Los autores agradecen al Programa de Jóvenes Investigadores del Departamento Administrativo de Ciencia, Tecnología e Innovación COLCIENCIAS, por la financiación de este trabajo de investigación, a través de la convocatoria 617 de 2013. A los productores caucheros, quienes facilitaron sus plantaciones, para el desarrollo de este estudio. Conflictos de intereses: El manuscrito fue preparado y revisado con la participación de todos los autores, quienes declaramos que no existe conflicto de intereses que ponga en riesgo la validez de los resultados presentados. Financiación: Este estudio fue financiado Departamento Administrativo de Ciencia, Tecnología e Innovación COLCIENCIAS y la Universidad de la Amazonia. 


\section{BIBLIOGRAFÍA}

1. ACOSTA, M.; VARGAS H., J.J.; VELÁZQUEZ M., A.Y.; ETCHEVERS B., J. 2002. Estimación de la biomasa aérea mediante el uso de relaciones alométricas en seis especies arbóreas en Oaxaca, México. Agrociencia. 36:725-736.

2. ALMEIDA, A.A.; GOMES, F.P.; ARAUJO, R.P.; SANTOS, R.C.; VALLE, R. 2014. Leaf gas exchange in species of the Theobroma genus. Photosynthetica. 52(1):1621.

3. AKIMA, H. 2015. Package 'akima'. Interpolation of irregularly spaced data. Disponible desde internet en: http://cran.r-project.org/web/ packages/akima/ akima.pdf (con acceso 04/01/2015).

4. AZCÓN, J.; FLECK, I.; ARANDA, X.; GÓMEZ, N. 2008. Fotosíntesis: factores ambientales y cambio climático. Fundamentos de Fisiología Vegetal. En: Azcón-Bieto, J.; Talón, M. (eds). McGraw-Hill Interamericana. Madrid. p.247-263.

5. CERDÁN, C.R. 2007. Conocimiento local sobre servicios ecosistémicos de cafeticultores del Corredor Biológico Volcán Central Talamanca, Costa Rica. Ambientico (Costa Rica). 166:3-7.

6. DaMATTA, F.M.; RODRÍGUEZ, N. 2007. Producción sostenible de cafetales en sistemas agroforestales del Neotrópico: una visión agronómica y ecofisiológica. Agr. Col. 25(1):113-123.

7. DaMATTA, F.M. 2004. Ecophysiological constraints on the production of shaded and unshaded coffee: a review. Field Crops Res. 86:99-114.

8. FARFÁN, F.; ARIAS, J.; RIAÑO, N. 2003. Desarrollo de una metodología para medir sombrío en sistemas agroforestales con café. Cenicafé 54(1):24-30.

9. GREENWOOD, M.S.; DAY, M.E.; BERLYN, G.P. 2009. Regulation of foliar plasticity in conifers: developmental and environmental factors. J Sustain. For. 28:48-62.

10. JAIMEZ, R.; TEZARA, W.; CORONEL, I.; URICH, R. 2008. Ecofisiología del cacao (Theobroma cacao): su manejo en el sistema agroforestal. Sugerencias para su mejoramiento en Venezuela. Rev. Forestal Venezolana. 52(2):253-258.
11. JARAMILLO-R., A.; AREILA-P., J.; MONTOYA-R., E.; QUÍROGA-Z, F.; 2006: La radiación solar; consideraciones para su estudio en las plantaciones de café (Coffea arabica L.). Meteorol. Col. 10:12-22.

12. KUME, A.; NASAHARA, K.N.; NAGAI, S.; MURAOKA, H. 2011. The ratio of transmitted near-infrared radiation to photosynthetically active radiation (PAR) increases in proportion to the adsorbed PAR in the canopy. J. Plant Res. 124(1):99-106.

13. LINDQUIST, J.L.; ARKEBAUER, J.T.; WALTERS, T.D.; CASSMAN, G.K.; DOBERMANN, A. 2005. Maize radiation use efficiency under optimal growth conditions. Agron. J. 97(1):72-78.

14. MARTÍNEZ, G.E.R.; MARTÍNEZ, A.V.; MATA, J.J.; HERNÁNDEZ, J.V.; LÓPEZ, D.J.P. 2005. Biomasa aérea en plantaciones comerciales de hule (Hevea brasiliensis Müll. Arg.) en el estado de Oaxaca, México. Agrociencia. 39(4):449-458.

15. NINEMETS, Ü.; ANTEN, N. 2009. Packing the Photosynthetic Machinery: From Leaf to Canopy. En: Laisk, A.; Nedbal, L.; Govindjee. Advences in photosynthesis and respiration. Ed. Govindjee (U.S.A.). 29(16):1-514.

16. NOCK, C.A.; CASPERSEN, J.P.; THOMAS, S.C. 2008. Large ontogenetic declines in intra-crown leaf area index in two temperate deciduous tree species. Ecology. 89:744-753.

17. OURADO-NETO, D.; FAVARIN, J.L. 2008. Radiation availability in agroforestry system of coffee and rubber trees. In: Jose, S.; Gordon, A.M (eds). Toward Agroforestry Design: An Ecological Approach. Springer Science. (Netherlands). 312p.

18. RIGHI, C; LUNZ, A; BERNARDES, M; PEREIRA, C; DOURADO, D; FAVARIN, J. 2008. Radiation availability in agroforestry system of coffee and rubber trees. In: Jose, S.; Gordon, A.M. (eds). Toward Agroforestry Design: An Ecological Approach. Springer. 312p.

19. SEGURA, M.; ANDRADE, H.J. 2007. Cómo construir modelos alométricos de volumen, biomasa o carbono de especies leñosas perennes? How to develop biomass models of woody perennials species. Agroforest. Américas (CATIE). 46:89-96.

20. SOMARRIBA, E.; BEER, J. 2011. Productivity of Theobroma cacao agroforestry systems with timber 
or legume service shade trees. Agroforest. Syst. 81(2):109-121.

21. STERLING C., A.; RODRÍGUEZ C., D.F.; HERNÁNDEZ R, C.; SALAS T., Y.M.; MAZORRA V., A.; BETANCURT P., B.; DUSSAN H., I.; GONGORA O., H. 2014. Clones promisorios de caucho en asocio con copoazú y plátano hartón con potencial para la amazonia colombiana. En: Introducción. Sterling, A; Rodríguez, C.H. (eds). Agroforestería en el Caquetá. Editorial Scripto S.A.S. Florencia. (Colombia). p.1762.

22. TALBOT, G.; DUPRAZ, C. 2012. Simple models for light competition within agroforestry discontinuous tree stands: are leaf clumpiness and light interception by woody parts relevant factors? Agrofor. Syst. 84(1):101-116.

23. TSUBO, M.; MUKHALA, E.; OGINDO, H.; WALKER, S. 2003. Productivity of maize-bean intercropping in a semi-arid region of South Africa. Water SA. 29(4):381-388.

24. TSUBO, M.; WALKER, S. 2005. Relationships between photosynthetically active radiation and clearness index at Bloemfontein, South Africa. Theoret. Appl. Climatol. 80(1):17-25.
25. VINCÉNT, G.; HARJA, D.; ASMARA, D. 2002. SLIM software: a simple light interception model for multi-species, multi-strata forests. Bois Forêts des Tropiques. 2(272):97-100.

26. WILSON, T.B.; MEYERS, T.P. 2007. Determining vegetation indices from solar and photosynthetically active radiation fluxes [J]. Agricult. Forest Meteorol. 144(3):160-179.

27. WU, G.; LEEUW, J.; SKIDMORE, A.; LIU, Y.; PRINS, H. 2010. Comparison of extrapolation and interpolation methods for estimating daily photosynthetically active radiation (PAR). Geo-spatial Inf. Sci. 13(4):235-242.

28. YUNLIN, Z.; BOQIANG, Q. 2002. The basic characteristic and climatological calculation of the photosythetically available radiation in Taihu region. Acta Energiae Solaris Sinica. 23(1):118-123.

29. ZHANG, X.; ZHANG, Y.; ZHOUB, Y. 2000. Measuring and modelling photosynthetically active radiation in Tibet Plateau during April-October. Agricult. Forest Meteorol. 102(2):207-212.

Recibido: Marzo 24 de 2015

Aceptado: Agosto 5 de 2015

\section{Cómo citar:}

Durán-Bautista, E.H.; Ángel-Sánchez, Y.K.; Suárez-Salazar, J.C. 2015. Dinámica de la radiación fotosintéticamente activa en arreglos agroforestales con Hevea brasiliensis en el norte de la Amazonia Colombiana. Rev. U.D.C.A Act. \& Div. Cient. 18(2): 365-372. 